\title{
SELECÃO DE GENÓTIPOS PARENTAIS DE ACEROLA COM BASE NA DIVERGÊNCIA GENÉTICA MULTIVARIADA ${ }^{1}$
}

\author{
VALÉRIA CARPENTIERI-PÍPOLO², DEONISIO DESTRO³, CÁSSIO EGIDIO CAVENAGHI PRETE ${ }^{3}$, \\ MARIA GLÓRIA NILO GONZALES ${ }^{4}$, IRENE POPPER ${ }^{5}$, SILVIA ZANATTA ${ }^{6}$ e FLÁVIO ANDRÉ MARTINS DA SILVA ${ }^{7}$
}

\begin{abstract}
RESUMO - Este trabalho teve por objetivo identificar e selecionar genótipos parentais de acerola (Malpighia emarginata L.) adequadas a programas de melhoramento genético. Nove caracteres quantitativos de maior importância agronômica foram usados para determinação da distância genética e formação de grupos similares de acessos. O agrupamento pelo método de Tocher, a partir das distâncias generalizadas de Mahalanobis, possibilitou a divisão de 14 genótipos em três grupos. Com base na divergência genética e no caráter agronômico-chave (teor de vitamina C), destacaram-se como mais promissores os cruzamentos dos genótipos: AM Mole pertencente ao grupo III, com os genótipos PR AM, № 18, PR 17, PR 16, Eclipse, AM 22 e Dominga, todos pertencentes ao grupo I.
\end{abstract}

Termos para indexação: Malpighia emarginata, características agronômicas, clones, distância genética, melhoramento de plantas, métodos de melhoramento.

\section{WEST INDIAN CHERRY PARENTAL GENOTYPE SELECTION BASED ON MULTIVARIATE GENETIC DIVERGENCE}

\begin{abstract}
The objective of this study was to evaluate genotypes toward the identification of superior West Indian Cherry (Malpighia emarginata L.) parents that produce high-performing progenies. Parents clones were evaluated in a completely randomized design with three replications and each plot consisted of three plants. Fourteen genotypes of West Indian Cherry were characterized in Londrina, PR, Brazil. Nine quantitative characters of bigger agronomic interest were used for determination of genetic distance and identification of similar groups among the genotypes. The grouping by the method, based on Mahalanobis generalized distance, made it possible to organize the 14 genotypes in three groups. The genetic divergence based on a key agronomic character (level of vitamin C) allowed to recommend the cross as of the following genotypes: amarela mole (group III) with genotypes PR AM, № 18, PR 17, PR 16, Eclipse, AM 22 and Dominga all of them group I.
\end{abstract}

Index terms: Malpighia emarginata, agronomic characters, genotypes, clones, genetic distance, plant breeding, breeding methods.

${ }^{1}$ Aceito para publicação em 18 de janeiro de 2000.

${ }^{2}$ Eng. Agrôn., D.Sc., Prof. Associado, Dep. de Agronomia, Universidade Estadual de Londrina (UEL), Caixa Postal 6001, CEP 86051-990 Londrina, PR.

E-mail: pipolo@uel.br

${ }^{3}$ Eng. Agrôn., D.Sc., Dep. de Agronomia, UEL.

E-mail: ddestro@uel.br, cassio@uel.br

${ }^{4}$ Eng. Agrôn., M.Sc., Prof. Adjunto, Dep. de Agronomia, UEL.

${ }^{5}$ Farmacêutica Bioquímica, Prof. Adjunto, Dep. de Tecnologia de Alimentos e Medicamentos, UEL.

${ }^{6}$ Eng. Agrôn, mestranda da Faculdade de Ciências Agronômicas, Universidade Estadual Paulista (UNESP), Caixa Postal 1237, CEP 18603-970 Botucatu, SP.

${ }^{7}$ Aluno do curso de Engenharia Agronômica, UEL. E-mail: flaviomartins@ hotmail.com.br

\section{INTRODUÇÃO}

A acerola (Malpighia emarginata L.), também conhecida como cereja-das-antilhas, destaca-se dentre as demais frutíferas pela composição de seus frutos, que possuem alto teor de vitamina C. Cem gramas de polpa de acerola pode conter até $5.000 \mathrm{mg}$ de ácido ascórbico (vitamina C). Esse conteúdo chega a ser cem vezes superior ao da laranja, ou dez vezes maior do que o da goiaba, as duas tidas como as frutas de mais alto conteúdo de vitamina C (Gonzaga Neto \& Soares, 1994). Em virtude de tal característica, é conferida a essa fruta um inestimável valor farmacológico e alimentício, por sua importância 
como alimento e por consistir em mais uma alternativa de fonte de vitamina $\mathrm{C}$ a baixo custo.

Dependendo do local e da população de insetos, pode ocorrer na aceroleira tanto autogamia quanto alogamia, sendo que a polinização cruzada é responsável pelo maior tamanho dos frutos (Couceiro,1985; Gonzaga Neto \& Soares, 1994).

Em um programa de melhoramento genético envolvendo hibridação, uma das etapas fundamentais é a eleição dos genótipos parentais com bom desempenho e ampla base genética. Uma alternativa para a escolha dos genótipos parentais é a análise do seu comportamento isolado e do resultado dos cruzamentos dialélicos. Porém, a necessidade de realização de grande número de cruzamentos manuais e a condução de experimentos envolvendo um grande número de híbridos limitam a utilização dos cruzamentos dialélicos. Assim, medidas da divergência genética obtidas antes que qualquer cruzamento seja realizado podem auxiliar os melhoristas a concentrar seus esforços nas combinações mais promissoras. A heterose, manifestada nos cruzamentos, está diretamente relacionada à divergência genética entre seus progenitores (Falconer, 1981).

Nascimento Filho et al. (1992) utilizaram o método de divergência genética para avaliar plantas jovens de guaranazeiro (Paullinia cupana var. sorbilis). De um total de 36 clones de guaraná em relação a 25 caracteres, os genótipos foram separados em seis grupos similares e selecionados oito genótipos para obtenção de gerações segregantes a partir de cruzamentos biparentais ou múltiplos.

Paiva (1994) avaliou a divergência genética de seringueira (Hevea spp.) com relação aos componentes principais de oito e sete caracteres no primeiro e segundo ano de idade da planta, respectivamente. Os dados revelaram que os dois primeiros componentes foram suficientes para explicar mais de $80 \%$ da variância observada nos dois anos. $\mathrm{O}$ autor não observou correspondência entre as observações do primeiro e do segundo ano, e concluiu ser necessário maior período de tempo para a avaliação das plantas. As técnicas de divergência genética entre os clones foram potencialmente viáveis para uso em programa de melhoramento de seringueira.

Para identificar genótipos parentais adequados a um programa de melhoramento genético de guandu
(Cajanus cajan L. Millsp), Pípolo et al. (1995) avaliaram o comportamento de 25 genótipos com relação a oito caracteres. Os genótipos foram, por eles, divididos em cinco grupos, e, considerando os caracteres altura da planta, a porcentagem de proteína e a porcentagem de óleo, recomendaram 24 cruzamentos, $8 \%$ do total dos cruzamentos possíveis, envolvendo os 25 genótipos avaliados.

Amaral Júnior et al. (1996) usaram o método de Tocher para estimar a diversidade genética entre genótipos de moranga (Cucurbita maxima Duch). Os autores indicaram as combinações mais promissoras, e discriminaram os caracteres morfoagronômicos mais importantes na caracterização da variabilidade. Com este procedimento estabeleceram quatro grupos e recomendaram quatro combinações de cruzamentos visando ampliar a base genética da espécie.

Com o objetivo de estimar a diversidade genética entre acessos de acerola, Souza (1996) utilizou dados enzimáticos e agronômicos para a caracterização de 16 acessos de acerola. O autor aplicou sete sistemas enzimáticos, e observou seis características agronômicas. As estimativas de divergência por este procedimento não apresentaram correlação significativa com as características agronômicas. Os 16 acessos foram agrupados nos sistemas isoenzimáticos em quatro grupos e os caracteres agronômicos, em três grupos. Para fins de melhoramento, a partir dos resultados, os autores sugeriram cruzamentos entre os três acessos com melhores desempenhos agronômicos.

$\mathrm{Na}$ cultura da aceroleira, a pesquisa tem-se intensificado na estimativa da produtividade dos clones e na caracterização nutricional e qualitativa dos frutos. Em média, a produtividade de $30 \mathrm{~kg} /$ planta/ano é considerada satisfatória nas condições do norte do Paraná, e teores de vitamina C acima de $1.000 \mathrm{mg}$ por $100 \mathrm{~g}$ de polpa atendem aos padrões desejados pela indústria, embora o nível mínimo exigido de vitamina $C$ para a comercialização no mercado internacional seja acima de $1.200 \mathrm{mg}$ por $100 \mathrm{~g}$ de polpa (Kawati, 1995; Bliska \& Leite 1995).

Este trabalho teve como objetivo identificar e selecionar genótipos parentais de acerola adequados a programas de melhoramento genético. 


\section{MATERIAL E MÉTODOS}

Quatorze clones de acerola, pertencentes à coleção da Fazenda-Escola da Universidade Estadual de Londrina, e procedentes de pomares comerciais do Norte do Paraná, foram avaliados quanto a nove caracteres agronômicos e bromatológicos no período de janeiro de 1996 a junho de 1997. O delineamento experimental utilizado foi o de blocos inteiramente ao acaso, com três repetições e cada parcela foi formada por três plantas.

Os clones avaliados são os seguintes: № 8 (1), IA 6 (2), № 7 (3), PR AM (4), № 18 (5), PR 17 (6), IA 21 (7), PR 16 (8), Eclipse (9), PR 24 (10), AM 22 (11), № 3 (12), AM Mole (13), Dominga (14).

Os caracteres avaliados foram: CF: comprimento do fruto $(\mathrm{mm})$ medido com paquímetro em três amostras de 15 frutos cada; DF: diâmetro do fruto $(\mathrm{mm})$, medido com o paquímetro em três amostras de 15 frutos cada; $\mathrm{PF}$ : peso do fruto $(\mathrm{g})$, avaliado em frutos maduros a partir do peso médio de três amostras com 15 frutos cada; PP: peso da polpa (g), avaliado em três amostras com 15 frutos maduros cada, de que se fez a extração da semente; PS: peso da semente $(\mathrm{g})$, determinado a partir do peso médio de três amostras, com 45 sementes cada; RP: rendimento da polpa (\%), obtido por meio da relação entre peso da polpa e o peso do fruto; VC: vitamina C (mg de ácido ascórbico/ $100 \mathrm{~g}$ de polpa), determinado em polpa macerada, com 2,6 diclorofenol-indofenol; AC: acidez ( $\mathrm{mL} \mathrm{NaOH}$ $1 \mathrm{~N} / 100 \mathrm{~g}$ ), determinado a partir da neutralização da acidez da polpa da fruta, por titulação com $\mathrm{NaOH} 0,1 \mathrm{~N}$; Brix $\left({ }^{\circ}\right)$, determinado com o refratômetro em amostras de frutos maduros (Association of Official Analytical Chemists, 1970).

Os valores da característica rendimento da polpa (RP), expressos em porcentagem (\%), foram transformados para arco seno da raiz quadrada da proporção (arc sen $\sqrt{\mathrm{x} / 100}$ ) conforme Steel \& Torrie (1980).

Além das análises de variância multivariadas e comparação das médias pelo critério proposto por Scott \& Knott (1974), foram obtidos ainda: a) estimativa da distância generalizada de Mahalanobis; b) estabelecimento de grupos similares, pelo método de otimação, proposto por Tocher, citado por Rao (1952). Exemplo pormenorizado desse método é apresentado por Singh \& Chaudhary (1977); c) análise gráfica da divergência, por meio de variáveis canônicas. Com esse procedimento, foram obtidas informações sobre a distância genética, por meio da dispersão dos escores de cada genótipo em sistema cartesiano, cujos eixos são estabelecidos pelo primeiro par canônico (Rao, 1952).

As correlações fenotípicas $\left(r_{F}\right)$, genotípicas $\left(r_{G}\right)$ e de ambiente $\left(r_{A}\right)$ foram calculadas entre todos os caracteres, usando as seguintes fórmulas (Falconer, 1981):

$\mathrm{r}_{\mathrm{F}}=\operatorname{COV}_{\mathrm{F}(\mathrm{x}, \mathrm{y})} /\left(\delta_{\mathrm{Fx}}^{2} \cdot \delta^{2} \mathrm{Fy}\right)^{0.5}$

$\mathrm{r}_{\mathrm{G}}=\operatorname{COV}_{\mathrm{G}(\mathrm{x}, \mathrm{y})} /\left(\delta_{\mathrm{Gx}}^{2} \cdot \delta^{2}{ }_{\mathrm{Gy}}\right)^{0.5}$

$\mathrm{r}_{\mathrm{A}}=\mathrm{COV}_{\mathrm{A}(\mathrm{x}, \mathrm{y})} /\left(\delta^{2}{ }_{\mathrm{Ax}} \cdot \delta^{2}{ }_{\mathrm{Ay}}\right)^{0.5}$

onde: $\operatorname{COV}_{\mathrm{F}(\mathrm{x}, \mathrm{y})}, \operatorname{COV}_{\mathrm{G}(\mathrm{x}, \mathrm{y})}$ e $\operatorname{COV}_{\mathrm{A}(\mathrm{x}, \mathrm{y})}$ correspondem à covariância fenotípica, genotípica e de ambiente entre os caracteres x e y; $\delta^{2} \mathrm{Fx}, \delta^{2} \mathrm{Gx}$ e $\delta^{2} \mathrm{Ax}$ correspondem à variância fenotípica, genotípica e de ambiente do caráter $\mathrm{x}$; e $\delta^{2} \mathrm{Fy}$, $\delta^{2}{ }_{G y}$ e $\delta^{2}{ }_{A y}$ correspondem à variância fenotípica, genotípica e de ambiente do caráter y.

Para testar os valores das correlações, foi utilizado o teste t. O grau de liberdade utilizado para as correlações fenotípicas e genotípicas foi o número de genótipos menos dois (G.L. $=$ G-2 = 14-2 = 12). Na tabela, com esse grau de liberdade, os valores encontrados foram 0,532 e 0,661 para $5 \%$ e $1 \%$ de significância, respectivamente. No teste da correlação de ambiente foi utilizado o grau de liberdade do resíduo (26). Os valores encontrados foram 0,374 e 0,478 a $5 \%$ e $1 \%$ de significância, respectivamente (Cruz \& Regazzi, 1997).

\section{RESULTADOS E DISCUSSÃO}

As análises de variâncias apresentadas na Tabela 1 e as comparações das médias das nove características apresentadas na Tabela 2 mostram a existência de diferenças significativas entre as médias dos 14 genótipos de acerola em todos os caracteres avaliados, exceto em CF. Os coeficientes de determinação genotípica (b\%) foram elevados, ou seja, acima de $85 \%$. Esses resultados evidenciam uma situação bastante favorável ao melhoramento, sugerindo a possibilidade de discriminar com boa eficiência genótipos parentais superiores entre os 14 genótipos avaliados. Os coeficientes de variação foram baixos, variando de $4,55 \%$ a $18,51 \%$, apresentando-se em níveis aceitáveis em termo de precisão experimental para ensaios conduzidos no campo.

O método de agrupamento proposto por Tocher, citado por Rao (1952), a partir das distâncias generalizadas de Mahalanobis (1936), possibilitou a divisão dos 14 genótipos em três grupos. O grupo I envolveu 12 genótipos (PR17, IA21, № 8, IA6, AM22, Eclipse, $\mathrm{N}^{\mathrm{o}} 7, \mathrm{~N}^{\mathrm{0}} 3$, PR 16, PR AM, № 18 e Dominga) correspondendo a 86\% dos acessos estudados; o grupo II envolveu o genótipo PR 24 e o III envolveu o genótipo AM Mole, representando cada um desses grupos 7\% do total dos acessos. Os genótipos PR17e IA 21 foram os mais relacionados genetica- 
TABELA 1. Resultados da análise de variância de nove caracteres em 14 genótipos de acerola, com as respectivas médias, coeficientes de variação (CV) e coeficiente de determinação genotípica (b\%). UEL, Londrina, PR, ano agrícola 1995/1996'.

\begin{tabular}{|c|c|c|c|c|c|c|c|c|c|c|}
\hline \multirow{2}{*}{$\begin{array}{l}\text { Fonte de } \\
\text { variação }\end{array}$} & \multirow[t]{2}{*}{ GL } & \multicolumn{9}{|c|}{ Quadrado médio } \\
\hline & & $\mathrm{CF}$ & $\mathrm{DF}$ & $\mathrm{PF}$ & $\mathrm{PP}$ & PS & $\mathrm{RP}$ & Vit. C & $\mathrm{AC}$ & Brix \\
\hline Blocos & 2 & 4,10 & 0,98 & 0,50 & 0,01 & 0,19 & 0,01 & 91584,69 & 3,80 & 0,53 \\
\hline Genótipos & 13 & $24,04 * *$ & $21,17 * *$ & $9,06^{* *}$ & $5,47 * *$ & $1,25^{* *}$ & $0,02 * *$ & $1073413,1 * *$ & $19,52 * *$ & $11,15^{* *}$ \\
\hline Resíduos & 26 & 3,56 & 1,43 & 0,79 & 0,31 & 0,07 & 0,00 & 24015,43 & 1,30 & 0,22 \\
\hline Média & & 15,15 & 12,13 & 5,60 & 4,19 & 1,34 & 1,06 & 1392,92 & 14,50 & 10,23 \\
\hline $\mathrm{CV}(\%)$ & & 12,45 & 9,84 & 15,89 & 13,25 & 18,51 & 5,59 & 11,13 & 7,86 & 4,55 \\
\hline $\mathrm{b} \%$ & & 85,19 & 93,27 & 91,25 & 94,37 & 95,05 & 85,21 & 97,76 & 93,35 & 98,06 \\
\hline
\end{tabular}

${ }^{1}$ CF: comprimento do fruto (mm); DF: diâmetro do fruto (mm); PF: peso do fruto (g); PP: peso da polpa (g); PS: peso da semente (g); RP: rendimento da polpa (\%) (estimativa obtida a partir de dados transformados para arc sen $\sqrt{\mathrm{x} / 100}$, conforme Steel \& Torrie (1980)); Vit. C (mg/100 g); AC: acidez $(\mathrm{mL} \mathrm{NaOH} 1 \mathrm{~N} / 100 \mathrm{~g})$; Brix $\left({ }^{\circ}\right)$.

** Significativo a $1 \%$ de probabilidade pelo teste $\mathrm{F}$.

TABELA 2. Comparação entre médias de 14 genótipos de acerola em relação a nove caracteres avaliados. UEL, Londrina, PR, ano agrícola 1995/1996'.

\begin{tabular}{lrrrrrrrrr}
\hline Genótipo & \multicolumn{1}{c}{ Vit. C } & \multicolumn{1}{c}{ RP } & \multicolumn{1}{c}{ CF } & \multicolumn{1}{c}{ DF } & PF & PP & PS & AC & Brix \\
\hline $\mathrm{N}^{\mathrm{o}} 8$ & $741,50 \mathrm{D}$ & $78,61 \mathrm{~A}$ & $9,40 \mathrm{~B}$ & $8,53 \mathrm{D}$ & $2,89 \mathrm{~B}$ & $2,28 \mathrm{D}$ & $0,61 \mathrm{D}$ & $13,25 \mathrm{~B}$ & $11,30 \mathrm{~B}$ \\
$\mathrm{IA} 6$ & $1014,16 \mathrm{C}$ & $81,78 \mathrm{~A}$ & $15,80 \mathrm{~A}$ & $11,46 \mathrm{C}$ & $5,80 \mathrm{~B}$ & $4,76 \mathrm{~B}$ & $1,03 \mathrm{D}$ & $14,05 \mathrm{~B}$ & $9,53 \mathrm{C}$ \\
$\mathrm{N}^{\mathrm{o}} 7$ & $1101,50 \mathrm{C}$ & $76,95 \mathrm{~A}$ & $16,46 \mathrm{~A}$ & $11,93 \mathrm{C}$ & $6,66 \mathrm{~B}$ & $5,16 \mathrm{~B}$ & $1,49 \mathrm{C}$ & $11,76 \mathrm{C}$ & $8,00 \mathrm{C}$ \\
PR AM & $2117,50 \mathrm{~A}$ & $62,33 \mathrm{~B}$ & $11,33 \mathrm{~B}$ & $8,26 \mathrm{D}$ & $4,42 \mathrm{~B}$ & $2,52 \mathrm{D}$ & $0,90 \mathrm{D}$ & $14,90 \mathrm{~B}$ & $9,06 \mathrm{C}$ \\
$\mathrm{N}^{\mathrm{o}}$ 18 & $2404,33 \mathrm{~A}$ & $84,78 \mathrm{~A}$ & $16,81 \mathrm{~A}$ & $13,69 \mathrm{~B}$ & $7,39 \mathrm{~B}$ & $3,74 \mathrm{C}$ & $0,64 \mathrm{D}$ & $17,36 \mathrm{~A}$ & $9,66 \mathrm{C}$ \\
PR 17 & $1241,00 \mathrm{C}$ & $76,10 \mathrm{~A}$ & $14,53 \mathrm{~A}$ & $12,20 \mathrm{C}$ & $5,01 \mathrm{~B}$ & $3,83 \mathrm{C}$ & $1,17 \mathrm{C}$ & $14,57 \mathrm{~B}$ & $11,90 \mathrm{~B}$ \\
IA 21 & $1198,00 \mathrm{C}$ & $79,43 \mathrm{~A}$ & $14,46 \mathrm{~A}$ & $10,20 \mathrm{C}$ & $4,35 \mathrm{~B}$ & $3,42 \mathrm{C}$ & $0,98 \mathrm{D}$ & $13,16 \mathrm{~B}$ & $10,70 \mathrm{~B}$ \\
PR 16 & $1435,00 \mathrm{~B}$ & $65,21 \mathrm{~B}$ & $18,60 \mathrm{~A}$ & $15,73 \mathrm{~A}$ & $8,55 \mathrm{~A}$ & $5,64 \mathrm{~B}$ & $2,90 \mathrm{~A}$ & $11,19 \mathrm{C}$ & $10,06 \mathrm{C}$ \\
Eclipse & $1607,50 \mathrm{~B}$ & $76,10 \mathrm{~A}$ & $16,13 \mathrm{~A}$ & $14,06 \mathrm{~B}$ & $5,54 \mathrm{~B}$ & $4,21 \mathrm{C}$ & $1,33 \mathrm{C}$ & $17,20 \mathrm{~A}$ & $8,93 \mathrm{C}$ \\
PR 24 & $470,50 \mathrm{D}$ & $76,10 \mathrm{~A}$ & $18,86 \mathrm{~A}$ & $17,40 \mathrm{~A}$ & $9,57 \mathrm{~A}$ & $7,28 \mathrm{~A}$ & $2,29 \mathrm{~B}$ & $15,42 \mathrm{~A}$ & $9,03 \mathrm{C}$ \\
AM 22 & $1564,50 \mathrm{~B}$ & $73,49 \mathrm{~A}$ & $14,93 \mathrm{~A}$ & $10,80 \mathrm{C}$ & $5,11 \mathrm{~B}$ & $3,75 \mathrm{C}$ & $1,35 \mathrm{C}$ & $17,20 \mathrm{~A}$ & $8,93 \mathrm{C}$ \\
$\mathrm{N}^{\mathrm{o}} 3$ & $614,50 \mathrm{D}$ & $86,18 \mathrm{~A}$ & $18,60 \mathrm{~A}$ & $14,53 \mathrm{~B}$ & $6,23 \mathrm{~B}$ & $5,41 \mathrm{~B}$ & $0,81 \mathrm{D}$ & $11,17 \mathrm{C}$ & $9,20 \mathrm{C}$ \\
AM Mole & $1740,83 \mathrm{~B}$ & $70,80 \mathrm{~B}$ & $11,73 \mathrm{~B}$ & $10,45 \mathrm{C}$ & $4,95 \mathrm{~B}$ & $3,55 \mathrm{C}$ & $1,37 \mathrm{C}$ & $19,11 \mathrm{~A}$ & $15,83 \mathrm{~A}$ \\
Dominga & $2250,00 \mathrm{~A}$ & $61,36 \mathrm{~B}$ & $14,46 \mathrm{~A}$ & $10,60 \mathrm{C}$ & $4,94 \mathrm{~B}$ & $3,05 \mathrm{D}$ & $1,89 \mathrm{~B}$ & $17,51 \mathrm{~A}$ & $9,20 \mathrm{C}$ \\
\hline
\end{tabular}

${ }_{1}$ Médias seguidas de letras diferentes na vertical divergem a 1\% de probabilidade pelo teste Scott \& Knott (1974); CF: comprimento do fruto (mm); DF: diâmetro do fruto (mm); PF: peso do fruto (g); PP: peso da polpa (g); PS: peso da semente (g); RP: rendimento da polpa (\%); Vit.C (mg/100 g); AC: acidez (mL NaOH $1 \mathrm{~N} / 100 \mathrm{~g})$; $\mathrm{Brix}\left({ }^{\circ}\right)$.

mente, sendo a distância entre eles a de menor magnitude $\left(\mathrm{D}^{2}=15,76\right)$. Os genótipos mais divergentes geneticamente foram PR 24 e AM Mole, com a maior distância observada $\left(\mathrm{D}^{2}=698,86\right)$. Diversos autores recomendam a utilização de genótipos parentais com a maior divergência possível para maximizar a heterose manifestada nos híbridos, aumentar a probabilidade de ocorrência de segregantes superiores em gerações avançadas e ampliar a base genética. Portanto, essas informações devem ser utilizadas para recomendação de cruzamentos. Tendo-se por base a estatística multivariada, espera-se encontrar alto grau de similaridade entre os genótipos pertencentes a um mesmo grupo. Dessa forma, deve-se evitar cruzamentos dentro de um mesmo grupo (Destro, 1991).

A identificação de genótipos com base somente na divergência genética, sem considerar seus próprios desempenhos, pode não ser uma boa estratégia para um programa de melhoramento. Assim, a recomendação de cruzamentos em programas de melhoramento entre genótipos divergentes, mas que apresentem desempenho superior nas principais características de importância agronômica, parece ser a mais indicada. 
Na quantificação da divergência genética entre os genótipos parentais, tem sido utilizada, tanto a técnica da conglomeração baseada na distância generalizada ( $\left.\mathrm{D}^{2}\right)$ de Mahalanobis (1936), quanto a análise das variáveis canônicas. No último caso, a divergência genética é avaliada pela dispersão gráfica dos escores relativos aos genótipos estudados em eixos cartesianos. Em geral, os pesquisadores têm optado pela representação gráfica, quando as duas primeiras variáveis canônicas estiverem acima de $70 \%$ da variabilidade total disponível (Cruz, 1990).

Nesta pesquisa, a dispersão presente na Fig. 1 foi feita em relação às duas primeiras variáveis canônicas, que retiveram $75 \%$ da variação total, sendo satisfatório para este estudo. As médias dos 14 genótipos de acerola nos 9 caracteres estudados encontram-se na Tabela 2.

A recomendação dos cruzamentos foi baseada no parâmetro referencial mínimo do produto no mercado externo, isto é, o conteúdo de vitamina $\mathrm{C}$ acima de $1.200 \mathrm{mg}$ por $100 \mathrm{~g}$ de polpa (Bliska \& Leite, 1995). Os cruzamentos recomendados foram os seguintes: do genótipo AM Mole (grupo III) com os genótipos
PR AM, No 18, PR16, Eclipse, AM 22 e Dominga (grupo I). Dessa maneira, seriam feitos sete cruzamentos, representando apenas $7,7 \%$ do total que poderia ser realizado no dialelo parcial entre os 14 genótipos avaliados, os quais permitiriam a obtenção de até 91 combinações.

As estimativas de correlações podem ser úteis quando determinado caráter de interesse é de difícil avaliação. Se esse caráter apresenta correlações fenotípicas e genotípicas significativas com outro de mais fácil acesso, pode-se fazer a seleção indireta com base no caráter de fácil acesso. Quando dois caracteres apresentam correlação positiva e significativa, a seleção em um resulta na melhoria do outro. Dificuldades surgem quando dois caracteres apresentam correlação positiva e significativa e um deles é indesejável, ou quando os dois caracteres são desejáveis, mas a correlação é negativa e significativa.

As correlações fenotípicas, genotípicas e de ambiente estão apresentadas na Tabela 3 .

As correlações fenotípicas e genotípicas positivas encontradas para acidez e Vitamina C de 0,565 e

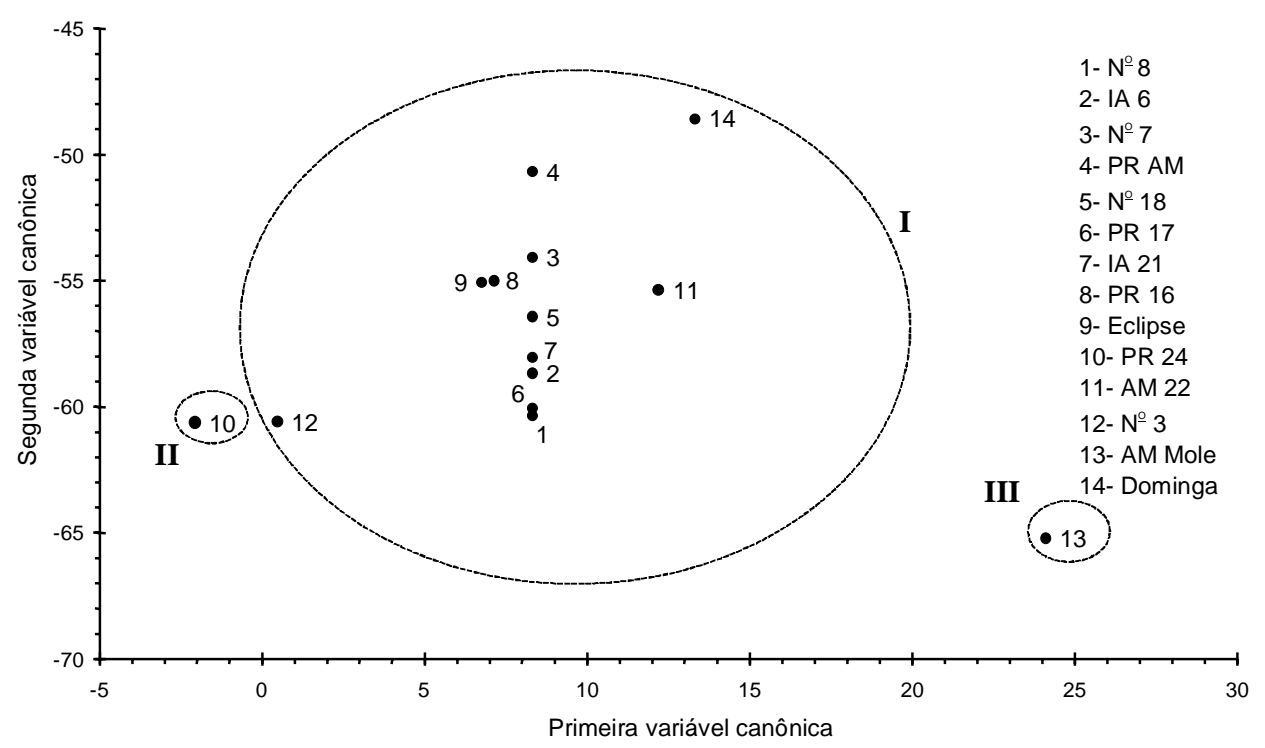

FIG . 1. Dispersão dos escores de 14 genótipos de acerola em relação às duas primeiras variáveis canônicas. 
TABELA 3. Estimativas dos coeficientes de correlação fenotípica (acima da diagonal), genotípica (acima da

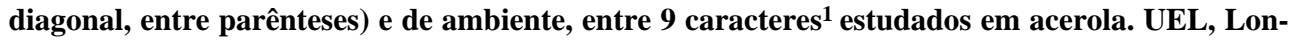
drina, PR, ano agrícola 1995/1996.

\begin{tabular}{|c|c|c|c|c|c|c|c|c|c|}
\hline Caráter & $\mathrm{CF}$ & DF & $\mathrm{PF}$ & $\mathrm{PP}$ & PS & $\mathrm{RP}^{3}$ & $\mathrm{VC}$ & $\mathrm{AC}$ & Brix \\
\hline $\mathrm{CF}$ & & $\begin{array}{r}0,899^{2} \\
(0,960)\end{array}$ & $\begin{array}{c}0,792 \\
(0,806)\end{array}$ & $\begin{array}{c}0,862 \\
(0,884)\end{array}$ & $\begin{array}{c}0,491 \\
(0,496)\end{array}$ & $\begin{array}{c}0,307 \\
(0,394)\end{array}$ & $\begin{array}{c}-0,241 \\
(-0,265)\end{array}$ & $\begin{array}{c}-0,285 \\
(-0,340)\end{array}$ & $\begin{array}{c}-0,505 \\
(-0,559)\end{array}$ \\
\hline $\mathrm{DF}$ & 0,438 & & $\begin{array}{c}0,836 \\
(0,862)\end{array}$ & $\begin{array}{c}0,889 \\
(0,912)\end{array}$ & $\begin{array}{c}0,562 \\
(0,564)\end{array}$ & $\begin{array}{c}0,257 \\
(0,032)\end{array}$ & $\begin{array}{c}-0,314 \\
(-0,332)\end{array}$ & $\begin{array}{c}-0,096 \\
(-0,112)\end{array}$ & $\begin{array}{c}-0,310 \\
(-0,328)\end{array}$ \\
\hline $\mathrm{PF}$ & 0,720 & 0,528 & & $\begin{array}{c}0,942 \\
(0,951)\end{array}$ & $\begin{array}{c}0,799 \\
(0,814)\end{array}$ & $\begin{array}{c}-0,083 \\
(-0,021)\end{array}$ & $\begin{array}{c}-0,391 \\
(-0,416)\end{array}$ & $\begin{array}{c}-0,247 \\
(-0,266)\end{array}$ & $\begin{array}{c}-0,321 \\
(-0,341)\end{array}$ \\
\hline PP & 0,761 & 0,530 & 0,844 & & $\begin{array}{c}0,587 \\
(0,588)\end{array}$ & $\begin{array}{c}0,250 \\
(0,285)\end{array}$ & $\begin{array}{c}-0,546 \\
(-0,571)\end{array}$ & $\begin{array}{c}-0,288 \\
(-0,309)\end{array}$ & $\begin{array}{c}-0,334 \\
(-0,346)\end{array}$ \\
\hline PS & 0,527 & 0,546 & 0,610 & 0,565 & & $\begin{array}{c}-0,555 \\
(-0,581)\end{array}$ & $\begin{array}{c}-0,060 \\
(-0,058)\end{array}$ & $\begin{array}{c}-0,087 \\
(-0,102)\end{array}$ & $\begin{array}{c}-0,101 \\
(-0,114)\end{array}$ \\
\hline $\mathrm{RP}^{3}$ & $-0,195$ & $-0,268$ & $-0,568$ & $-0,06$ & $-0,372$ & & $\begin{array}{c}-0,458 \\
(-0,508)\end{array}$ & $\begin{array}{c}-0,211 \\
(-0,235)\end{array}$ & $\begin{array}{c}-0,010 \\
(-0,098)\end{array}$ \\
\hline $\mathrm{VC}$ & 0,006 & 0,069 & 0,041 & 0,070 & $-0,123$ & 0,105 & & $\begin{array}{c}0,565^{* *} \\
(0,583)^{* *}\end{array}$ & $\begin{array}{c}0,083 \\
(0,096)\end{array}$ \\
\hline $\mathrm{AC}$ & 0,178 & 0,140 & $-0,004$ & 0,037 & 0,146 & $-0,015$ & 0,197 & & $\begin{array}{c}0,365 \\
(0,038)\end{array}$ \\
\hline BRIX & 0,116 & 0,099 & 0,021 & 0,045 & 0,291 & 0,203 & 0,522 & 0,112 & \\
\hline
\end{tabular}

0,583, respectivamente, indicaram que a seleção de um desses dois caracteres pode ser feita por meio apenas da seleção daquele que apresentar maior facilidade na avaliação (Tabela 3).

As correlações fenotípicas e genotípicas positivas e elevadas $(0,799$ e 0,814 , respectivamente $)$ entre peso da semente e peso do fruto indicam que frutos de maior peso apresentaram sementes maiores. Portanto, a seleção de plantas com frutos grandes e sementes pequenas é bastante dificultada (Tabela 3 ).

Os caracteres avaliados referentes a tamanho do fruto (comprimento e diâmetro) resultaram em correlações fenotípicas e genotípicas positivas com o peso da polpa, apresentando o diâmetro do fruto os maiores valores das correlações $(0,889$ e 0,912 , respectivamente) (Tabela 3 ) .
As correlações fenotípicas e genotípicas do peso da polpa e peso do fruto apresentaram altos valores positivos $(0,942$ e 0,951 , respectivamente), o que indica seguramente que a seleção de frutos grandes (de maior diâmetro) resulta em maiores quantidades de polpa (Tabela 3).

\section{CONCLUSÕES}

1. Neste estudo de divergência genética, é possível dividir os 14 genótipos em 3 grupos: o grupo I encerra 12 genótipos, o que corresponde a $86 \%$ dos genótipos; os grupos II e III envolvem um genótipo cada, representando $7 \%$ dos genótipos.

2. Tendo como base o estudo da divergência ge- 
nética e o caráter agronômico mais importante (Vitamina C) os cruzamentos recomendados são os seguintes: genótipo AM Mole (grupo III) com os genótipos PR AM, № 18, PR 17, PR 16, Eclipse, AM 22 e Dominga (grupo I), obtendo-se 7 cruzamentos, totalizando $7,7 \%$ do total que poderia ser feito; dessa maneira, são feitos apenas 7 cruzamentos do total de 91, sem incluir os recíprocos, que podem ser realizados no dialelo parcial entre os 14 genótipos avaliados.

3. A seleção de frutos grandes com sementes pequenas é dificultada por causa das correlações fenotípica e genotípica positivas entre os caracteres peso de fruto e peso de semente.

4. A seleção de frutos grandes com maior diâmetro resulta na seleção de frutos com maior quantidade de polpa.

\section{REFERÊNCIAS}

AMARAL JÚNIOR, A.T.; CASALI, V.W.D.; CRUZ, C.D.; FINGER, F.L. Utilização de variáveis canônicas e de análise de agrupamentos na avaliação da divergência genética entre acesso de moranga. Horticultura Brasileira, Brasília, v.14, n.2, p.182$184,1996$.

ASSOCIATION OF OFFICIAL ANALYTICAL CHEMISTS (Arlington, Estados Unidos). Official methods of analysis. 11.ed. Washington, 1970. $1015 p$.

BLISKA, F.M.M.; LEITE, R.S.S.F. Aspectos econômicos e mercado. In: SÃO JOSÉ, A R.; ALVES, R.E. Acerola no Brasil: produção e mercado. Vitória da Conquista : UESB, 1995.160p.

COUCEIRO, E.M. Curso de extensão sobre a cultura da acerola. Recife : UFRPE, 1985. 45p.

CRUZ, C.D. Aplicações de algumas técnicas multivariadas no melhoramento de plantas. Piracicaba : ESALQ, 1990. 188p. Tese de Doutorado.

CRUZ, C.D.; REGAZZI, A.J. Modelos biométricos aplicados ao melhoramento genético. Viçosa : UFVImprensa Universitária, 1997. 390p.

DESTRO, D. Capacidade de combinação de genótipos de soja (Glycine max (L.) Merrill) apropriados para o consumo humano. Piracicaba : ESALQ, 1991. 158p. Tese de Doutorado.

FALCONER, D.S. Introduction to quantitative genetics. 2.ed. London : Longman, 1981. 340p.

GONZAGA NETO, L.; SOARES, J.M. Acerola para exportação: aspectos técnicos da produção. Brasília : Embrapa-SPI, 1994. 43p. (Publicações Técnicas Frupex, 10).

KAWATI, R. Pesquisa e extensão sobre a cultura da acerola no Estado de São Paulo. In: SÃO JOSÉ, A R.; ALVES, R.E. Acerola no Brasil: produção e mercado. Vitória da Conquista : UESB, 1995. 160p.

MAHALANOBIS, P.C. On the generalized distance in statistics. National Academy of Sciences, India Proceedings, Allahabad, v.2, p.49-55, 1936.

NASCIMENTO FILHO, F.J.; CRUZ, C.D.; GARCIA, T.B. Divergência genética em plantas jovens de guaranazeiro e possibilidades de melhoramento. Pesquisa Agropecuária Brasileira, Brasília, v.27, n.12, p.1571-1577, dez. 1992.

PAIVA, J.R. Divergência genética entre clones primários de seringueira. Pesquisa Agropecuária Brasileira, Brasília, v.29, n.4, p.607-615, abr. 1994.

PÍPOLO, V.C.; PÍPOLO, A.E.; DESTRO, D.; GUERRA, E.P. Seleção de genótipos de guandu baseada na divergência genética multivariada. Pesquisa Agropecuária Brasileira, Brasília, v.30, n.7, p.977982, jul. 1995.

RAO, R.C. Advanced statistical methods in biometric research. New York: J. Wiley, 1952. 390p.

SCOTT, A.J.; KNOTT, M.A. A cluster analysis method for grouping means in the analysis of variance. Biometrics, Washington, v.30, p.507-512, 1974.

SINGH, R.K.; CHAUDHARY, B.D. Biometrical methods in quantitative genetic analysis. New Delhi : Kalyani, 1977. 304p.

SOUZA, J.C. Diversidade genética entre acessos de acerola (Malpighia sp.) com base em dados isozimáticos e agronômicos, Viçosa : UFV, 1996. 67p. Dissertação de Mestrado.

STEEL, R.G.; TORRIE, J.H. Principles and procedures of statistics. 2.ed. New York : McGraw-Hill, 1980. $633 p$.

Pesq. agropec. bras., Brasília, v.35, n.8, p.1613-1619, ago. 2000 